\title{
Low-Loss Electric and Magnetic Field-Enhanced Spectroscopy with Subwavelength Silicon Dimers
}

Pablo Albella, ${ }^{*}, \dagger, \S$ M. Ameen Poyli, ${ }^{\dagger}$ Mikolaj K. Schmidt, ${ }^{\dagger}$ Stefan A. Maier, ${ }^{\ddagger}$ Fernando Moreno,"

Juan José Sáenz, ${ }^{\perp}$ and Javier Aizpurua* ${ }^{\dagger}$

${ }^{\dagger}$ Centro de Física de Materiales CFM (CSIC-UPV/EHU), and Donostia International Physics Center (DIPC), P. Manuel de

Lardizabal 5, 20018 San Sebastián, Spain,

${ }^{\ddagger}$ Experimental Solid State Group, Department of Physics, Imperial College London, London SW7 2AZ, U.K.

${ }^{\S}$ Optical and Semiconductor Devices Group, Department of Electrical and Electronic Engineering, Imperial College London, London SW7 2AZ, U.K.

"Grupo de Óptica, Departamento de Física Aplicada, Universidad de Cantabria, Avenida de los Castros s/n, 39005 Santander, Spain

${ }^{\perp}$ Departamento de Física de la Materia Condensada and Instituto Nicolás Cabrera and Condensed Matter Physics Center (IFIMAC), Universidad Autónoma de Madrid, 28049 Madrid, Spain

ABSTRACT: Dielectric nanoparticles with moderately high refractive index and very low absorption (like $\mathrm{Si}$ and Ge in the visible-near-infrared (VIS-NIR) range) show a magnetodielectric behavior that produces interesting far-field coherent effects, like directionality phenomena or field enhancement in the proximity of the particle surface. As in the case of metals, ensembles of two or more dielectric particles can constitute basic elements for developing new spectroscopic tools based on surface field enhancement effects. Here we explore the electromagnetic behavior of the basic unit constituted by a dimer of dielectric nanoparticles made of moderately low-loss high refractive index material. The interactions responsible for the spectral features of the scattered radiation and field enhancement of the dimer are identified and studied through an analytical dipole-dipole model. The fluorescence of a single emitter (either electric or magnetic dipole) located in the dimer gap is also explored by
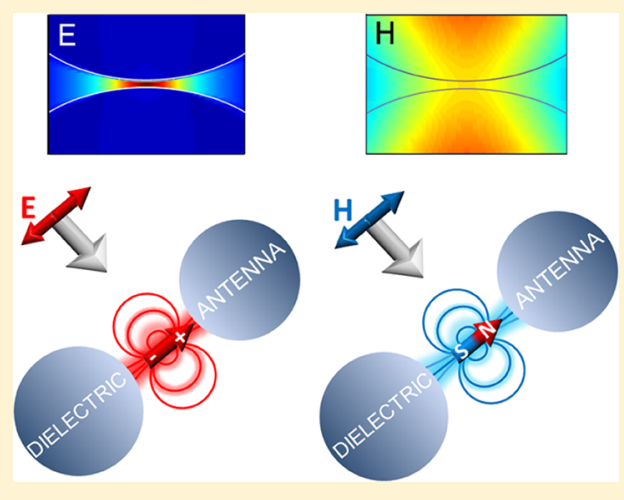
calculating the quantum efficiencies and the quenching/enhancement of the radiation rates. Along this analysis, a comparison with metallic dimers is carried out. This study opens new possibilities to perform field-enhanced spectroscopy and sensing with nanostructures made of suitable dielectric materials.

\section{INTRODUCTION}

Nanoscale structures are fundamental building blocks to control and engineer the electromagnetic behavior of light at subwavelength dimensions. Metallic nanostructures are an object of high interest due to their ability to show resonances in the optical response given by the collective behavior of the conduction electrons near the surface of the metal, the so-called surface plasmons. These resonances depend strongly on the optical properties and geometry of the structures, allowing for the manipulation of light at subwavelength dimensions, thus acting as optical nanoantennas and providing a versatile tool to control light beyond the conventional diffraction limit. ${ }^{1}$ However, plasmonic excitations are known to be affected by relatively large losses. This unavoidable problem has stimulated the study of subwavelength dielectric particles with high permittivity, ${ }^{2,3}$ providing a potential to constitute building blocks of novel photonic platforms dealing with optical magnetism. ${ }^{4,5,6}$ In particular, silicon submicrometer-sized spherical particles show interesting scattering properties ${ }^{7,8,9}$ in the near-infrared range of the spectrum whose magnetic response has been experimentally demonstrated recently. ${ }^{4}$
Unlike plasmonic antennas ${ }^{10}$ and phononic antennas, ${ }^{11}$ where antenna effects are produced by the oscillations of the free electron plasma and collective lattice vibrations, respectively, dielectric antennas rely on the fields and displacement currents induced in the antenna structure. Furthermore, dielectric antennas can also provide control of the far-field radiation properties of nearby emitters due to the properties of coherence of the radiation of electric and magnetic modes. ${ }^{12-14}$ This coherent admixture of both types of modes can produce control over directionality of the scattered radiation, as shown theoretically ${ }^{15-17}$ and experimentally, for both microwaves ${ }^{18}$ and visible light. ${ }^{19,20}$ Other remarkable aspects of the interaction between high refractive index particles concern the possibilities to induce optical forces ${ }^{15,21,22}$ and to take advantage of near-field interactions for single-particle spectroscopy. $^{23}$

Received: March 18, 2013

Revised: May 13, 2013

Published: May 17, 2013 
Here, we show that low-loss dielectric nanoantennas can be a powerful alternative to their plasmonic counterparts as an element to control the optical response at the nasnoscale. To this end, we exploit the coherent interaction between the electric and magnetic dipolar resonances induced in subwavelength low-loss high refractive index spherical particles. We analyze a system composed by a pair of spheres interacting in close proximity as a natural combination that provides straightforward field enhancement and confinement derived from the response of the single-particle situation. This is probably the simplest realistic problem of multiple scattering by finite bodies, and it has been extensively studied in other fields like colloidal, aerosol, or atmosphere science, analytical chemistry, photonic crystals, astrophysics, etc. ${ }^{24-27}$ However, most of the earlier work focused on either small Rayleigh particles or large spheres showing high-order sharp resonances without strong overlap between the magnetic and electric responses. We present the results of numerical calculations of the far-field scattering properties and the near-field enhancement for a dimer of subwavelength silicon spheres. A simple analytical model, based on standard multiple scattering theory, ${ }^{24}$ shows how the mutual interactions of the electric and magnetic dipolar modes driven at each particle lead to the enhanced scattering obtained. Unlike the case of metals, the low-loss nature of silicon, its abundancy in nature, its lower conductivity, and its better heat resistivity are encouraging features that drive this study. We also explore the potential of these dielectric nanoparticles as effective antennas to produce enhancement of the fluorescence of single emitters such as molecules or quantum dots located in their proximity. ${ }^{8,28,29}$ Indeed, loss-less Mie particles have been proven to promote magnetic transitions in atoms or ions. ${ }^{30}$ Such processes are usually enhanced due to a 2-fold aspect: enhancement of the excitation rate due to concentration of light by the antennas ${ }^{31,32}$ and enhancement of the emission rate from the molecules in the so-called Purcell effect. ${ }^{33,34}$ We will discuss here the effect of placing electric and magnetic dipolar emitters in the gap of the dimer, modifying the emission rates and the quantum efficiencies of such emitters. We will show that, while dielectric and plasmonic antennas provide comparable enhancements for the radiative decay rate of dipolar emitters, silicon nanoantennas yield larger and more stable quantum efficiencies since, unlike in plasmonic dimers, the energy of the dipolar emitters is not dissipated by Ohmic losses.

This paper is organized as follows: Section 2 is devoted to analyze the near- and far-field enhancement achieved when a couple of subwavelength magnetodielectric silicon spherical particles forming a dimer interact with optical electromagnetic radiation. This configuration will be compared with conventional metallic antenna arrangements made of gold. Section 3 presents a numerical model based on the calculation of the interaction between two electromagnetic units, each one composed of both electric and magnetic dipoles. This will serve to analyze the different contributions to the electromagnetic interaction between the spheres. Section 4 analyzes the fluorescence enhancement properties of electric and magnetic dipolar emitters located in the gap of a silicon sphere dimer. A comparison with the results from a more conventional metallic dimer will be also presented. Finally, a summary and the main conclusions of this research will be drawn.

\section{FIELD ENHANCEMENT IN A PAIR OF SUBWAVELENGTH SILICON SPHERES}

When moderately high refractive index dielectric nanoparticles are excited with light, the electromagnetic field is highly concentrated inside the material due to generation of intense displacement currents. This effect produces inner electric field distributions which, for resonant frequencies, lead to electric and/or magnetic dipolar responses to the incident radiation. In both cases, electric field distributions are also produced outside of the particle similar to those in the proximities of metallic nanoparticles when plasmon resonances are excited. ${ }^{35}$ Consequently, when dielectric nanoparticles are coupled to form dielectric antennas, they also provide field enhancement and localization of electromagnetic energy in the antenna gap. We thus analyze the electromagnetic properties of a dielectric antenna as depicted in Figure 1. It corresponds to a dimer of

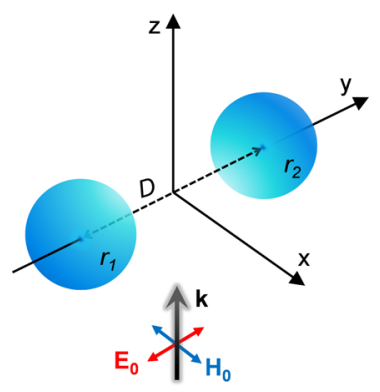

Figure 1. Schematic representation of a Si particle dimer showing the orientation of the incoming radiation and its electric and magnetic polarization (p-polarized). The size of each spherical particle is $r_{1}=r_{2}$ $=150 \mathrm{~nm}$, and the center to center separation is $D$.

silicon subwavelength spheres placed in close proximity. The size of each sphere is $150 \mathrm{~nm}$ in radius to keep their resonances in the near-infrared range of the spectrum. The optical constants for silicon and gold were obtained from Palik. ${ }^{36}$

Figure 2(a) shows the extinction spectra in one of such dimers for different separation distances. The system is illuminated by a plane wave with the wavevector perpendicular to the dimer axis and polarized along the dimer axis (see schematics in inset of Figure 2a). The extinction spectra of the system have been numerically calculated with the finite difference in time domain (FDTD) method. ${ }^{37}$ To identify the origin of each spectral feature, the spectral extinction of one single $\mathrm{Si}$ sphere is also shown (black dashed line) as a reference. In this case three peaks can be clearly identified for incident wavelengths of $\lambda \simeq 1150 \mathrm{~nm}, \lambda \simeq 864 \mathrm{~nm}$, and $\lambda \simeq 800 \mathrm{~nm}$, corresponding to magnetic dipolar, electric dipolar, and magnetic quadrupolar resonances, respectively. Although there is a partial overlap between the first dipolar peaks (magnetic-electric), the magnetic resonant peak is still very well resolved. Interestingly, for wavelengths larger than $\lambda \simeq 820$ $\mathrm{nm}$, the cross section is completely determined by the first Mie coefficients. In other words, in this regime Si particles can be treated as non-Rayleigh dipolar scatterers.

When two of these dielectric spheres are coupled together at close proximity, the electric and magnetic resonances interact, distorting and producing new spectral features. We observe that both the magnetic and the electric resonances are shifted (the magnetic resonance to the blue and the electric resonance to the red), and their intensities vary as the gap is reduced. Another interesting feature in the spectra is the appearance of 

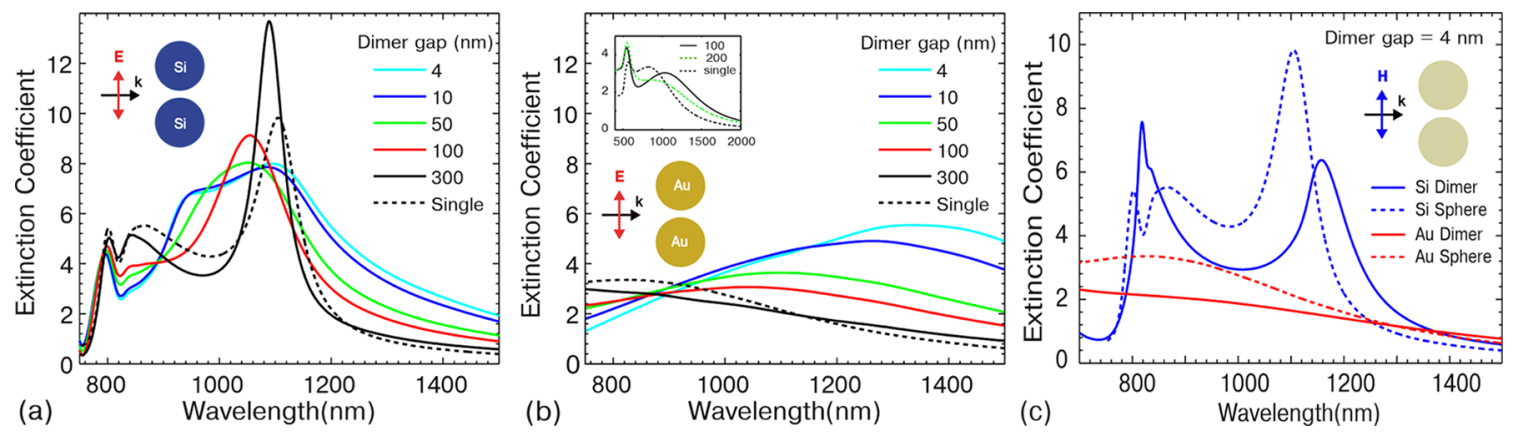

Figure 2. Extinction coefficient of (a) silicon and (b) gold dimers of spherical particles of radius $150 \mathrm{~nm}$ calculated for different separating distances of the gaps. The plane wave illumination propagates perpendicular to the dimer axis with electric field polarization parallel to the dimer axis ( $\mathrm{p}$ polarized), as shown in the inset. The top-left inset in (b) shows the spectra of the plasmonic dimer in a broader spectral range. (c) Extinction coefficient of silicon (blue solid line) and gold (red solid line) dimers with a $4 \mathrm{~nm}$ gap, illuminated with plane wave propagating perpendicular to the dimer axis and with s-polarized incident light as shown in the inset. Extinction of a single sphere of silicon and gold is shown in dashed lines as a reference.
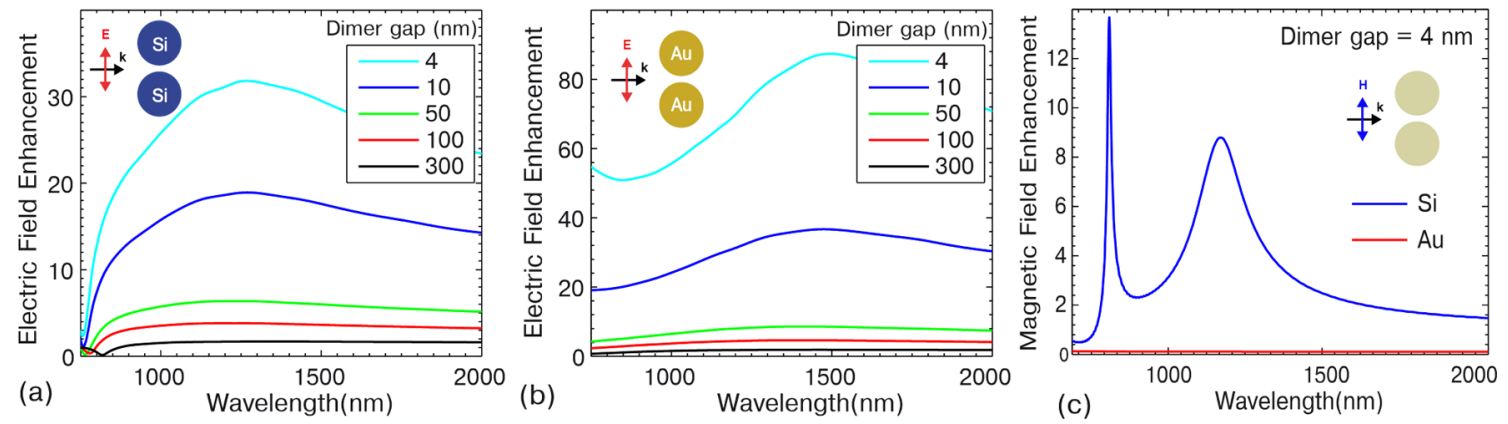

Figure 3. (a) Near-field enhancement of the electric field amplitude as a function of wavelength calculated at the center of the gap in a silicon dimer with different separation distances. (b) Same as in (a) but for a gold dimer. (c) Near-field enhancement of the magnetic field amplitude as a function of wavelength calculated at the center of the gap for a silicon dimer (blue line) and for a gold dimer (red line). (a) and (b) obtained for p-polarized incident light and (c) obtained for s-polarized incident light.

an intermediate peak around $\lambda \simeq 960 \mathrm{~nm}$ when the dimers get closer than $50 \mathrm{~nm}$. We will consider this point as the threshold separation to trigger what we will define as the strong interaction regime. The appearance of this new intermediate peak is accompanied with an unusual blue-shift of the magnetic dipolar resonance. The mutual interaction of the electric and magnetic dipolar resonances responsible for the enhanced near field and scattering in a broad spectral range will be discussed later, based on an electric-magnetic dipole-dipole interaction model.

To compare the far-field scattering by a silicon dimer with a typical metallic system used in field-enhanced spectroscopy, in Figure $2 \mathrm{~b}$ we show the extinction cross section of a gold dimer of the same size, under the same illumination conditions and gaps. The comparison shows that the silicon dimer scatters as much as the gold dimer in the near-infrared range even exceeding the values of the plasmonic dimer at resonance.

Figure 2c also shows a comparison of the extinction coefficient of a Si dimer (blue solid line) and a gold dimer (red solid line) when illuminated with s-polarized light, that is, with the magnetic field along the dimer axis. Extinction of single silicon and gold spheres is shown as a reference in dashed lines. Similar to the case of p-polarized excitation when two of these dielectric spheres are coupled together at close proximity, the electric and magnetic resonances interact, distorting and producing new spectral features. However, now we observe that it is the magnetic resonance that red shifts due to the reinforcing of aligned magnetic dipoles and the electric one that blue shifts as a result of interaction of parallel dipoles, ${ }^{38}$ the latter overlapping and fusing with the magnetic quadrupolar resonance. We also point out that no matter the incident polarization the silicon dimer offers a better far-field response in the near-infrared regime.

In addition to enhanced far-field scattering, field-enhanced spectroscopy also demands an intense and localized electric field. Calculations demonstrate that intense localized electric near fields can be achieved in the gap of a dielectric dimer for the arrangement shown in the inset of Figure 2a. Figure 3 shows the electric near-field enhancement calculated at the center of the gap of a silicon dimer (Figure 3a) and a gold dimer (Figure 3b), considering different gap widths. Unlike in the case of metals, the incident electric field creates a depolarizing field inside the dielectric sphere. At the dipolar resonance, this depolarizing field gives rise to an intense dipolar electric near-field distribution around the sphere. Similar to the case of metallic particles, when two of such dielectric spheres are brought together to form a dimer, the dipolar field distribution together with the nonradiative higher-order modes originate the intense electric near-field in the gap. This nearfield enhancement obtained in the case of dielectric antennas compared to that of plasmonic antennas, even though slightly smaller, is large enough to allow field-enhanced spectroscopy. It can be seen in the literature that average enhancement factors larger than $10^{4}-10^{5}$ may be quoted as SERS enhancements. ${ }^{39}$ Nevertheless, the slight decrease in the field enhancement obtained with dielectric antennas can be compensated by the 


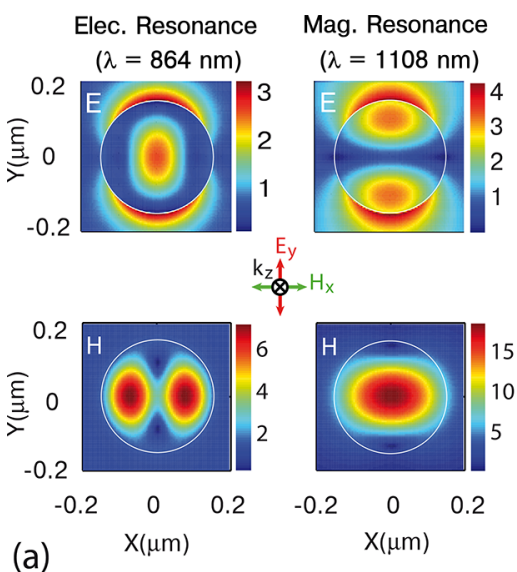

(a)

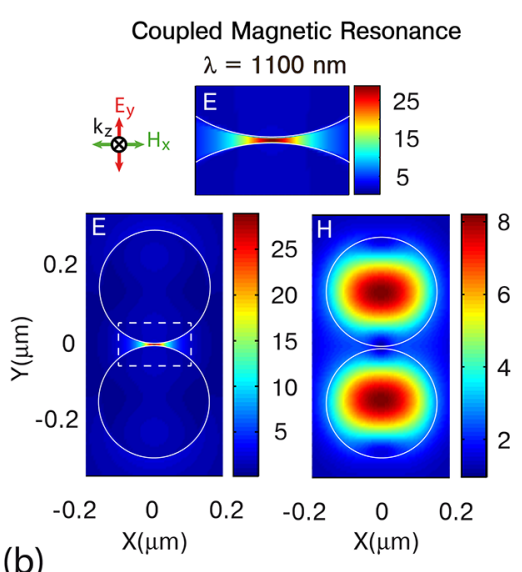

(b)

(C)

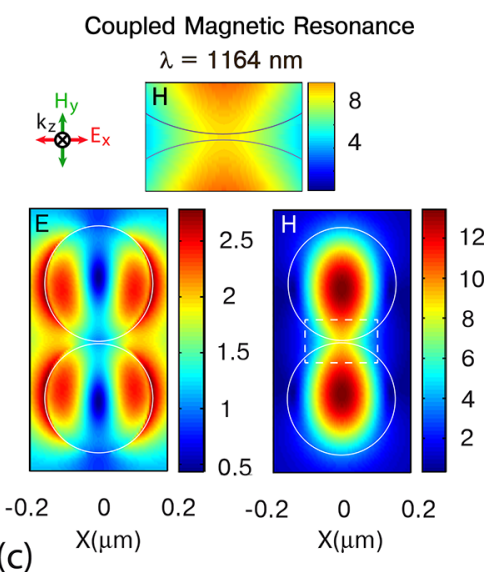

$\mathrm{X}(\mu \mathrm{m})$

Figure 4. (a) Near-field amplitude enhancement distribution near a silicon particle of $150 \mathrm{~nm}$ radius at the electric $(\lambda=864 \mathrm{~nm})$ and magnetic $(\lambda=$ $1108 \mathrm{~nm}$ ) dipolar resonances calculated in the plane normal to $k$ and parallel to the dimer axis (p-polarized). (b) Electric near-field enhancement $(E /$ $\left.E_{\text {inc }}\right)$ and magnetic near-field enhancement $\left(H / H_{\text {inc }}\right)$ calculated for a dimer of radius $150 \mathrm{~nm}$ and dimer gap $4 \mathrm{~nm}$ at $\lambda=1100 \mathrm{~nm}$ for incident electric field polarized along the dimer axis. The area marked with a dashed square is zoomed in on top of the figure showing the electric field enhancement. The field distribution clearly shows the localization of the enhanced electric field in the dimer gap. (c) Electric and magnetic near-field enhancements calculated for the same system as in (b) for incident magnetic field polarized along the dimer axis (s-polarized). The area marked with a dashed line is zoomed in on top of the figure showing the magnetic field enhancement. This configuration helps to extract the enhanced magnetic field to the dimer gap.

absence of absorption at the particles and a larger scattering cross section, a situation of interest in field-enhanced fluorescence for example.

In Figure 3c we compare the magnetic field in the gap of a $\mathrm{Si}$ dimer (blue line) and a Au dimer (red line), showing how the $\mathrm{Si}$ dimer can also effectively enhance the magnetic field when the incident light is s-polarized, that is, with the incident magnetic field parallel to the dimer axis. This excitation boosts the coupling of the magnetic modes supported by the close spheres forming the dimer. This is an interesting result, showing how low-loss dielectric dimers can enhance the electric, the magnetic, or both fields depending on the incident polarization ( $p$-polarized, s-polarized, or circularly polarized).

The electric and magnetic near-field distributions in the proximity of an isolated submicrometer silicon sphere at resonant wavelengths are discussed in detail in ref 7 . Here in Figure 4 we compare the electric and magnetic near-field distributions of a single silicon sphere with that of a dimer. The sphere radius is $150 \mathrm{~nm}$ for both cases, and the gap between spheres forming the dimer is $4 \mathrm{~nm}$. Figure $4 \mathrm{a}$ shows the electric and magnetic near-field map of the single sphere at the electric and magnetic resonance wavelengths calculated in the plane normal to $k$ and parallel to the dimer axis. Under plane wave illumination, the scattering from the $150 \mathrm{~nm}$ silicon sphere can be studied using Mie theory. The lowest-energy Mie resonance (magnetic dipolar resonance) in the Mie expansion is a magnetic dipole. Physically this corresponds to a situation where the electric field inside the sphere creates a circular electric alternate current loop, which mimics the behavior of a magnetic dipole. The current loop and the resultant dipolar magnetic field distribution can be identified by observing the near-field maps in Figure 4a calculated at magnetic resonance $(\lambda=1108 \mathrm{~nm})$. At the second lowest-energy Mie resonance (the electric resonance), the depolarization field in the particle oscillates resonant to the incident field to create a dipolar electric field distribution around the sphere. This corresponds to the electric dipole, which is clear from the near-field maps calculated at the electric resonance $(\lambda=864 \mathrm{~nm})$ of the sphere in Figure 4a.
When two such spheres are brought together to form a dimer and the system is illuminated with a plane wave polarized parallel to the dimer axis, the interaction of the induced electric and magnetic dipoles gives rise to a field distribution as shown in Figure 4b. In addition to enhanced far-field scattering in a broad spectral range (Figure 2), the near-field spectra also show enhancement of the electric field in a broader range of the spectrum. The field map clearly shows the localization of the enhanced electric field in the dimer gap calculated at $\lambda=1100$ $\mathrm{nm}$, although similar maps are obtained on a broad spectral range. Under the dipole approximation, the intense field in the gap can be understood as a result of two interacting electric dipoles oriented in the same direction close to each other. Since the induced magnetic dipoles in the dimer are arranged parallel and side by side, the net effect is to reduce the strength of the induced magnetic moment in each sphere when they are brought close to each other to form the dimer. This clearly explains why the magnetic near-field enhancement in a silicon dimer is lower than that compared to the single sphere case. To achieve enhanced magnetic field in the dimer gap, we use a configuration with incident magnetic field parallel to the dimer axis. Figure $4 \mathrm{c}$ shows the electric and the magnetic near-field enhancement distribution calculated at the coupled magnetic resonance $(\lambda=1164 \mathrm{~nm})$ for this configuration. Complementary to the electric field enhancement in the antenna gap for the p-polarized incident light, a magnetic field enhancement is observed in the case of s-polarized incidence.

\section{ELECTRIC AND MAGNETIC DIPOLE-DIPOLE INTERACTION MODEL FOR A DIELECTRIC DIMER}

To understand the origin of the spectral features in the extinction spectra of the dimer and the evolution of the spectra with the gap size, it is convenient to understand the system based on a simple electrodynamical model of interaction. To that end, we propose a dipolar model to study the mutual interaction of the electric-electric, magnetic-magnetic, and electric-magnetic dipole pairs induced in the silicon spheres both in and out of resonance. With the help of this model we are able to reproduce the dimer scattering spectra and the 
evolution of the spectra for different dimer gaps. We also identify the different mutual electric and magnetic interactions contributing to the total spectral response.

Scattering from a Silicon Dimer. The system used to model the electromagnetic response of the silicon sphere dimer is based on the substitution of each particle by electric and magnetic dipoles, as shown in Figure 5. Two silicon spheres of

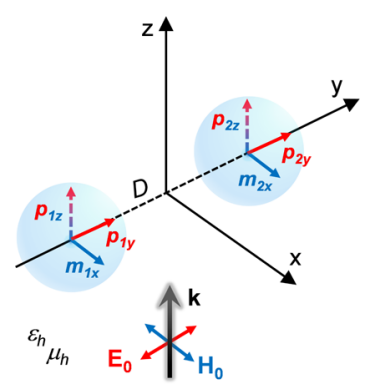

Figure 5. Schematic representation of the dipolar system used to model the electromagnetic response of the silicon sphere dimer. Red (blue) arrows on the spheres correspond to the induced electric (magnetic) dipoles. The electric dipoles along the $z$-axis (displayed with dashed lines) are the secondary dipoles induced by the field of the primary magnetic dipoles, and they are not excited directly by the incoming plane wave. The studied spherical area resembling the $\mathrm{Si}$ particles is substituted by a distribution of point dipoles $p_{1 y}, m_{1 x}, p_{1 z}$, $p_{2 y}, m_{2 x}$, and $p_{2 z}$.

radius $a$ are centered at $\mathbf{r}_{1}$ and $\mathbf{r}_{2}$, separated by a distance $D$ between the particle centers. The spheres are characterized by the complex dielectric function of silicon. They are illuminated with a monochromatic plane wave incident along $\mathrm{z}$ with the electric field $\mathbf{E}_{0}$ polarized along the dimer axis $(y)$. The dimer is immersed in an infinite homogeneous lossless medium with relative dielectric permittivity $\varepsilon_{h}$ and magnetic permeability $\mu_{h}$ (i.e., with refractive index $\left.n_{h}=\left(\varepsilon_{h} \mu_{h}\right)^{1 / 2}\right)$.

For plane-wave illumination, the electric and the magnetic fields incident on the dipoles are given by

$$
\begin{aligned}
& \mathbf{E}_{0}(\mathbf{r})=E_{0} e^{i k z} \hat{\mathbf{e}}_{y} \\
& \mathbf{H}_{0}(\mathbf{r})=-\frac{E_{0}}{Z} e^{i k z} \hat{\mathbf{e}}_{x} \equiv-H_{0} e^{i k z} \hat{\mathbf{e}}_{x}
\end{aligned}
$$

where $k$ is the wavenumber $k=\left(\varepsilon_{h} \mu_{h}\right)^{1 / 2} \omega / c\left(c=1 /\left(\varepsilon_{0} \mu_{0}\right)^{1 / 2}\right.$ is the speed of light in vacuum; $\varepsilon_{0}$ is the vacuum permittivity; and $\omega$ is the frequency), and $Z \equiv\left(\mu_{0} \mu_{h} /\left(\varepsilon_{0} \varepsilon_{h}\right)\right)^{1 / 2}$ is the vacuum impedance.

We assume here that subwavelength silicon spheres can be characterized by their electric and magnetic polarizabilities, $\alpha_{\mathrm{e}}=\left(6 \pi i / k^{3}\right) a_{1}$ and $\alpha_{\mathrm{m}}=\left(6 \pi i / k^{3}\right) b_{1}$, respectively, being $a_{1}$ and $b_{1}$ the electric and magnetic dipolar coefficients of the Mie expansion. ${ }^{40}$ This assumption is valid as long as we keep the size parameter, $x=k r \leq 1$. The total electric and magnetic fields at any point $r$ are given by the sum of the incident fields, $\mathbf{E}_{0}$ and $\mathbf{H}_{0}$, and the electric and magnetic field scattered by the two particles, $\mathbf{E}_{\text {scat }}$ and $\mathbf{H}_{\text {scat }}$ respectively.

$$
\begin{gathered}
\mathbf{E}_{\mathrm{scat}}(\mathbf{r})=\sum_{j=1,2}\left\{\frac{k^{2}}{\varepsilon_{0} \varepsilon_{h}} \overleftrightarrow{\mathbf{G}}_{\mathrm{E}}\left(\mathbf{r}-\mathbf{r}_{j}\right) \cdot \mathbf{p}_{j}+\right. \\
\left.i Z k^{2} \overleftrightarrow{\mathbf{G}}_{\mathrm{M}}\left(\mathbf{r}-\mathbf{r}_{j}\right) \cdot \boldsymbol{m}_{\mathbf{j}}\right\}
\end{gathered}
$$

$$
\begin{gathered}
\mathbf{H}_{\mathrm{scat}}(\mathbf{r})=\sum_{j=1,2}\left\{-i \frac{1}{Z} k^{2} \varepsilon_{0} \varepsilon_{h} \overleftrightarrow{\mathbf{G}}_{\mathrm{M}}\left(\mathbf{r}-\mathbf{r}_{j}\right) \cdot \mathbf{p}_{j}+\right. \\
\left.k^{2} \overleftrightarrow{\mathbf{G}}_{\mathrm{E}}\left(\mathbf{r}-\mathbf{r}_{j}\right) \cdot \boldsymbol{m}_{j}\right\}
\end{gathered}
$$

where $\stackrel{\leftrightarrow}{\mathbf{G}}_{\mathrm{E}}(\mathbf{r})$ and $\stackrel{\mathrm{G}}{\mathrm{M}}_{\mathrm{M}}(\mathbf{r})$ are the free-space electric and magnetic dyadic Green's functions, respectively ${ }^{35,41}$

$$
\begin{aligned}
\overleftrightarrow{\mathbf{G}}_{\mathrm{E}}(\mathbf{r}) \cdot \mathbf{p}= & \left\{\left(1+\frac{i}{k r}-\frac{1}{k^{2} r^{2}}\right) \mathbf{p}+\left(-1-\frac{3 i}{k r}+\frac{3}{k^{2} r^{2}}\right)\right. \\
& \left.\left(\boldsymbol{u}_{r} \cdot \mathbf{p}\right) \boldsymbol{u}_{r}\right\} g(r) \\
\overleftrightarrow{\mathbf{G}}_{\mathrm{M}}(\mathbf{r}) \cdot \mathbf{p}= & \left(\mathbf{u}_{\boldsymbol{r}} \times \mathbf{p}\right)\left(i-\frac{1}{k r}\right) g(r)
\end{aligned}
$$

$\mathbf{u}_{r}$ is the unit vector along $\mathbf{r}$ and $g(r)=e^{i k r} /(4 \pi r)$ is the scalar Green's function. Note that

$$
\overleftrightarrow{\mathbf{G}}_{\mathrm{M}} \equiv \frac{1}{k} \nabla \times \stackrel{\mathrm{G}}{\mathrm{E}}
$$

The induced dipoles, $\mathbf{p}_{j}$ and $\mathbf{m}_{j}$, on sphere " $j$ " are proportional to the corresponding incident total fields, $\mathbf{E}_{\text {inc }}\left(r_{j}\right)$ and $\mathbf{H}_{\text {inc }}\left(r_{j}\right)$, on each particle, respectively, being $j=1,2$

$$
\begin{aligned}
& \mathbf{p}_{j}=\varepsilon_{0} \varepsilon_{h} \alpha_{\mathrm{e}} \mathbf{E}_{\mathrm{inc}}\left(\mathbf{r}_{j}\right) \\
& \mathbf{m}_{\boldsymbol{j}}=\alpha_{\mathrm{m}} \mathbf{H}_{\mathrm{inc}}\left(\mathbf{r}_{j}\right)
\end{aligned}
$$

where $\alpha_{\mathrm{e}}$ and $\alpha_{\mathrm{m}}$ are the electric and magnetic polaribilities, respectively. ${ }^{40}$

Each of these induced dipoles will experience the field of the other dipole, and they will interact self-consistently to produce the actual dipole moments. Thus, induced dipoles moments are the solutions of the equations of self-consistently coupled dipoles

$$
\begin{gathered}
\mathbf{p}_{1}=\varepsilon_{0} \varepsilon_{h} \alpha_{\mathrm{e}} \mathbf{E}_{0}\left(\mathbf{r}_{1}\right)+\alpha_{\mathrm{e}} k^{2} \overleftrightarrow{\mathbf{G}}_{\mathrm{E}}\left(\mathbf{r}_{1}-\mathbf{r}_{2}\right) \cdot \mathbf{p}_{2}+ \\
i \varepsilon_{0} \varepsilon_{h} \alpha Z k^{2} \overleftrightarrow{\mathbf{G}}_{\mathrm{M}}\left(\mathbf{r}_{1}-\mathbf{r}_{2}\right) \cdot \mathbf{m}_{2} \\
\mathbf{m}_{1}=\alpha_{\mathrm{m}} \mathbf{H}_{0}\left(\mathbf{r}_{1}\right)-i \frac{\alpha \mathrm{m}}{Z} \frac{k^{2}}{\varepsilon_{0} \varepsilon_{h}} \overleftrightarrow{\mathbf{G}}_{\mathrm{M}}\left(\mathbf{r}_{1}-\mathbf{r}_{2}\right) \cdot \mathbf{p}_{2} \\
\quad+\alpha_{m} k^{2} \overleftrightarrow{\mathbf{G}}_{\mathrm{E}}\left(\mathbf{r}_{1}-\mathbf{r}_{2}\right) \cdot \mathbf{m}_{2} \\
\mathbf{p}_{2}=\varepsilon_{0} \varepsilon_{h} \alpha_{\mathrm{e}} \mathbf{E}_{0}\left(\mathbf{r}_{2}\right)+\alpha_{\mathrm{e}} k^{2} \overleftrightarrow{\mathbf{G}}_{\mathrm{E}}\left(\mathbf{r}_{2}-\mathbf{r}_{1}\right) \cdot \mathbf{p}_{1}+ \\
i \varepsilon_{0} \varepsilon_{h} \alpha_{\mathrm{e}} Z k^{2} \overleftrightarrow{\mathbf{G}}_{\mathrm{M}}\left(\mathbf{r}_{2}-\mathbf{r}_{1}\right) \cdot \mathbf{m}_{1} \\
\mathbf{m}_{2}=\alpha_{\mathrm{m}} \mathbf{H}_{0}\left(\mathbf{r}_{2}\right)-i \frac{\alpha_{\mathrm{m}}}{Z} \frac{k^{2}}{\varepsilon_{0} \varepsilon_{h}} \overleftrightarrow{\mathbf{G}}_{\mathrm{M}}\left(\mathbf{r}_{2}-\mathbf{r}_{1}\right) \cdot \mathbf{p}_{1} \\
\quad+\alpha_{\mathrm{m}} k^{2} \overleftrightarrow{\mathbf{G}}_{\mathrm{E}}\left(\mathbf{r}_{2}-\mathbf{r}_{1}\right) \cdot \mathbf{m}_{1}
\end{gathered}
$$

On the right-hand side of eq 10 the first term is related to the response to the incident electric field. The middle term is proportional to the action of the electric field originated by the electric dipole induced in the other particle labeled as " 2 ", and the last contribution is given by the induced magnetic field of the magnetic dipole induced in the particle labeled as " 2 ". In eq 11 the first term is related to the direct contribution of the incident magnetic field giving rise to an induced dipole moment. The middle term originates from the induced electric 
field of the other dipole, and the final term is the result of the magnetic field of the magnetic dipole on the other particle. Equations 12 and 13 follow the same pattern with the indexes 1 and 2 exchanged.

Solving eqs $10-13$ provides the values of the self-consistent electric and magnetic dipoles induced at each of the dimer spheres. Notice that this is a particular case of the formal solution of the two-sphere system ${ }^{25,26}$ considering only the two first $\left(a_{1}, b_{1}\right)$ coefficients in the Mie expansion of each sphere. Now the problem of the scattering dimer reduces to the calculation of the field scattered by the coherent superposition of the electric and magnetic dipoles obtained. The next section is devoted to the calculation of the total electric and magnetic field scattered by this set of dipoles representing the response of the dimer.

Electric Polarization of the Incoming Plane Wave along the Dimer Axis. To explain the spectra obtained from the simulations shown in Figure 2, we calculate the selfconsistent values of the electric and magnetic dipoles induced in the two silicon spheres. We use Green's function formalism to express the values of electric and magnetic fields at the position of the electric and magnetic dipoles in the two spheres located at $\mathbf{r}_{1}=(0,+D / 2,0)$ and $\mathbf{r}_{2}=(0,-D / 2,0)$ (i.e., the gap between the sphere surfaces is $d=D-2 a>0)$. The plane wave is incident along $z$ with the electric field polarized along the $y$ axis (dimer axis) and magnetic field along the $x$ axis as depicted in Figure 5. Then $\mathbf{r}_{1}-\mathbf{r}_{2}=D \hat{\mathbf{e}}_{y}$ and

$$
\begin{aligned}
& \overleftrightarrow{\mathbf{G}}_{\mathrm{E}}\left(\mathbf{r}_{1}-\mathbf{r}_{2}\right) \cdot \hat{\mathbf{e}}_{x}=\left\{\left(1+\frac{i}{k D}-\frac{1}{k^{2} D^{2}}\right)\right\} g(D) \hat{\mathbf{e}}_{x} \equiv-g_{x x} \hat{\mathbf{e}}_{x} \\
& \overleftrightarrow{\mathbf{G}}_{\mathrm{M}}\left(\mathbf{r}_{1}-\mathbf{r}_{2}\right) \cdot \hat{\mathbf{e}}_{x}=-\left(i-\frac{1}{k D}\right) g(D) \hat{\mathbf{e}}_{z} \equiv g_{z x} \hat{\mathbf{e}}_{z} \\
& \overleftrightarrow{\mathbf{G}}_{\mathrm{E}}\left(\mathbf{r}_{1}-\mathbf{r}_{2}\right) \cdot \hat{\mathbf{e}}_{z}=\left\{\left(1+\frac{i}{k D}-\frac{1}{k^{2} D^{2}}\right)\right\} g(D) \hat{\mathbf{e}}_{z} \equiv-g_{x x} \hat{\mathbf{e}}_{z} \\
& \overleftrightarrow{\mathbf{G}}_{\mathrm{M}}\left(\mathbf{r}_{1}-\mathbf{r}_{2}\right) \cdot \hat{\mathbf{e}}_{z}=\left(i-\frac{1}{k D}\right) g(D) \hat{\mathbf{e}}_{x} \equiv-g_{z x} \hat{\mathbf{e}}_{x} \\
& \overleftrightarrow{\mathbf{G}}_{\mathrm{E}}\left(\mathbf{r}_{1}-\mathbf{r}_{2}\right) \cdot \hat{\mathbf{e}}_{y}=\left\{-\frac{2 i}{k r}+\frac{2}{k^{2} D^{2}}\right\} g(D) \hat{\mathbf{e}}_{y} \equiv g_{y y} \hat{\mathbf{e}}_{y} \\
& \overleftrightarrow{\mathbf{G}}_{\mathrm{M}}\left(\mathbf{r}_{1}-\mathbf{r}_{2}\right) \cdot \hat{\mathbf{e}}_{y}=0
\end{aligned}
$$

Equations 14-19 allow for obtaining the $x, y$, and $z$ components of the electric and magnetic fields at the position of particle $2\left(\mathbf{r}_{2}\right)$, created by the dipoles located at particle 1 $\left(\mathbf{r}_{1}\right)$. The fields at $\mathbf{r}_{1}$ can be derived using the following relationships

$$
\begin{aligned}
& \overleftrightarrow{\mathbf{G}}_{\mathrm{E}}\left(\mathbf{r}_{2}-\mathbf{r}_{1}\right)=\overleftrightarrow{\mathbf{G}}_{\mathrm{E}}\left(\mathbf{r}_{1}-\mathbf{r}_{2}\right) \\
& \overleftrightarrow{\mathbf{G}}_{\mathrm{M}}\left(\mathbf{r}_{2}-\mathbf{r}_{1}\right)=-\overleftrightarrow{\mathbf{G}}_{\mathrm{M}}\left(\mathbf{r}_{1}-\mathbf{r}_{2}\right)
\end{aligned}
$$

When the incident light is polarized along $y$, i.e., along the dimer axis, the induced electric and magnetic dipole moments can be expressed as

$$
\begin{aligned}
& p_{1 y}=\varepsilon_{0} \varepsilon_{h} \alpha_{\mathrm{e}} E_{0}+\alpha_{\mathrm{e}} k^{2} g_{y y} p_{2 y} \\
& p_{2 y}=\varepsilon_{0} \varepsilon_{h} \alpha_{\mathrm{e}} E_{0}+\alpha_{\mathrm{e}} k^{2} g_{y y} p_{1 y}
\end{aligned}
$$

$$
\begin{aligned}
& p_{1 z}=-\alpha_{\mathrm{e}} k^{2} g_{x x} p_{2 z}+i \varepsilon_{0} \varepsilon_{h} \alpha_{\mathrm{e}} Z k^{2} g_{z x} m_{2 x} \\
& p_{2 z}=-\alpha_{\mathrm{e}} k^{2} g_{x x} p_{1 z}-i \varepsilon_{0} \varepsilon_{h} \alpha Z k_{\mathrm{e}}^{2} g_{z x} m_{1 x} \\
& m_{1 x}=-\frac{\alpha_{\mathrm{m}}}{Z} E_{0}+i \frac{\alpha_{\mathrm{m}}}{Z} \frac{k^{2}}{\varepsilon_{0} \varepsilon_{h}} g_{z x} p_{2 z}-\alpha_{\mathrm{m}} k^{2} g_{x x} m_{2 x} \\
& m_{2 x}=-\frac{\alpha_{\mathrm{m}}}{Z} E_{0}-i \frac{\alpha_{\mathrm{m}}}{Z} \frac{k^{2}}{\varepsilon_{0} \varepsilon_{h}} g_{z x} p_{1 z}-\alpha_{\mathrm{m}} k^{2} g_{x x} m_{1 x}
\end{aligned}
$$

The first term on the right-hand side of eqs 22 and 23 is the induced electric dipole by the incident electric field, and the second term is due to the induced electric dipole oriented along $y$ in the other sphere. In eqs 24 and 25, the first term is induced by the induced electric dipole in the neighboring particle and the second term by the magnetic dipole induced in the neighboring particle. The induced magnetic dipoles are oriented along the $x$ axis (transverse to the dimer axis) and show three contributions: the first contribution comes from the incident electric field, the middle term is induced by the induced electric dipole along $z$ in the neighboring sphere, and the final term is the effect of the neighboring magnetic dipole oriented along $x$. Now we define the dressed polarizabilities $\tilde{\alpha}$ as

$$
\begin{gathered}
\tilde{\alpha}_{\mathrm{e} y} \equiv \frac{\alpha_{\mathrm{e}}}{1-\alpha_{\mathrm{e}} k^{2} g_{y y}}, \\
\tilde{\alpha}_{\mathrm{e} z} \equiv \frac{\alpha_{\mathrm{e}}}{1-\alpha_{\mathrm{e}} k^{2} g_{x x}}, \\
\tilde{\alpha}_{\mathrm{m} x} \equiv \frac{\alpha_{\mathrm{m}}}{1+\alpha_{\mathrm{m}} k^{2} g_{x x}}
\end{gathered}
$$

From eqs 22 and 23, we obtain

$$
p_{1 y}=\frac{\varepsilon_{0} \varepsilon_{h} \alpha_{\mathrm{e}}\left(1+\alpha_{\mathrm{e}} k^{2} g_{y y}\right) E_{0}}{1-\alpha_{\mathrm{e}}^{2} k^{4} g_{y y}^{2}}
$$

Simplifying and substituting the dressed polarizability $\tilde{\alpha}_{\mathrm{e} y}$ from eq 28 gives

$$
p_{1 y}=\varepsilon_{0} \varepsilon_{h} \tilde{\alpha}_{\mathrm{e} y} E_{0}
$$

Following similar steps, substituting $p_{1 y}$ from eq 22 into eq 23 and comparing with eq 30 gives

$$
p_{1 y}=\varepsilon_{0} \varepsilon_{h} \tilde{\alpha}_{e y} E_{0}=p_{2 y}
$$

Similarly, from eq 24 to 27

$$
\begin{aligned}
& p_{1 z}=i \varepsilon_{0} \varepsilon_{h} \tilde{\alpha}_{\mathrm{e} z} Z k^{2} g_{z x} m_{2 x}=-p_{2 z} \\
& m_{1 x}=-\frac{\tilde{\alpha}_{\mathrm{m} x}}{Z} E_{0}+i \frac{\tilde{\alpha}_{\mathrm{m} x}}{Z} \frac{k^{2}}{\varepsilon_{0} \varepsilon_{h}} g_{z x} p_{2 z}=m_{2 x}
\end{aligned}
$$

In summary, we obtain the following set of self-consistent induced dipoles in particles 1 and 2

$$
\begin{aligned}
& p_{1 y}=p_{2 y}=\varepsilon_{0} \varepsilon_{h} \tilde{\alpha}_{\mathrm{e} y} E_{0} \\
& p_{1 z}=-p_{2 z}=-i \varepsilon_{0} \varepsilon_{h} E_{0} \frac{\tilde{\alpha}_{\mathrm{m} x} \tilde{\alpha}_{\mathrm{e} z} k^{2} g_{z x}}{1-\tilde{\alpha}_{\mathrm{m} x} \tilde{\mathrm{e}}_{\mathrm{e} z} k^{4} g_{z x}^{2}} \equiv-i \varepsilon_{0} \varepsilon_{h} E_{0} \tilde{\alpha}_{\mathrm{e}-\mathrm{m}}
\end{aligned}
$$


(a)

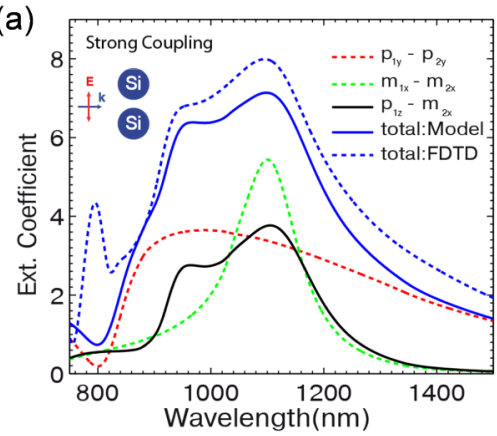

(b)

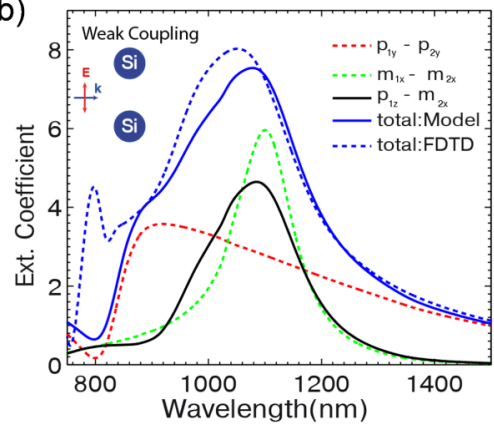

(c)

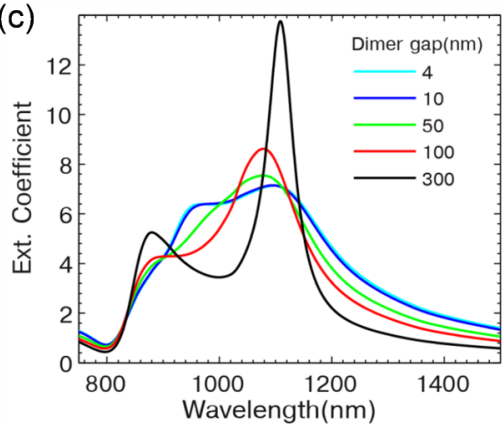

Figure 6. Extinction spectra of two interacting electric and magnetic dipoles calculated using the dipole-dipole model for strong (a) and weak (b) coupling regimes are compared with the FDTD results, for gap separations of 10 and $50 \mathrm{~nm}$, respectively. The model allows us to study the interactions responsible for the far-field spectra in Figure 2a by separating the contributions of each interaction. The blue dashed lines are the FDTD calculations shown in Figure 2a. (c) Extinction coefficient obtained for a broader range of dimer separations under the same dipole-dipole approximation used in (a) and (b). Gap distances are labeled in the inset.

$$
m_{1 x}=m_{2 x}=-\frac{\tilde{\alpha}_{\mathrm{m} x}}{Z} E_{0}-\left(\tilde{\alpha}_{\mathrm{e}-\mathrm{m}} k^{2} g_{z x}\right) \frac{\tilde{\alpha}_{\mathrm{m} x}}{Z} E_{0}
$$

Equations 28, 34, 35, and 36 summarize the self-consistent calculation of induced dipoles (see the corresponding distribution in Figure 5). Notice that $p_{1 y}$ only couples with $p_{2 y}$ (giving rise to the dressed polarizability $\tilde{\alpha}_{\text {ey }}$ ). However, the magnetic dipoles $m_{1 x}$ and $m_{2 x}$ are coupled both directly $\left(\tilde{\alpha}_{\mathrm{m} x}\right)$ and through the interaction with the induced electric dipoles along the $z$-axis (last term on the right-hand side of eq 36). The electric dipoles along $z$ are not excited directly by the incoming external field (eq 35) in the illumination considered here.

Scattering Amplitude Dyadic: Extinction Cross Section. For plane wave incidence with wavevector $\mathbf{k}_{\text {in }}$ and electrically polarized along $\hat{\mathbf{e}}_{\mathrm{in}}$, the electric and magnetic incident fields are given by

$$
\begin{aligned}
& \mathbf{E}_{0}(\mathbf{r})=E_{0} e^{i \mathbf{k}_{\text {in }} \cdot \mathbf{r}} \hat{\mathbf{e}}_{\text {in }} \\
& \mathbf{H}_{0}(\mathbf{r})=\frac{E_{0}}{Z} e^{i \mathbf{k}_{\text {in }} \cdot \mathbf{r}} \frac{\mathbf{k}_{\text {in }} \times \hat{\mathbf{e}}_{\text {in }}}{k}
\end{aligned}
$$

The scattered far-field can be written in terms of the vectorial scattering amplitude, $\mathcal{F}\left(\mathbf{k}_{\text {in }}, \mathbf{k}_{\text {out }}\right.$ ) (see ref 42 )

$$
\lim _{r \rightarrow \infty} \mathbf{E}_{\text {scat }}(\mathbf{r}) \approx E_{0} \frac{e^{i k r}}{r} \mathcal{F}\left(\mathbf{k}_{\text {in }}, \mathbf{k}_{\text {out }}\right) \cdot \hat{\mathbf{e}}_{\text {in }}
$$

The magnetic field is given by

$$
\mathbf{H}_{\text {scat }} \approx \frac{1}{k Z}\left(\mathbf{k}_{\text {out }} \times \mathbf{E}_{\text {scat }}\right)
$$

and the time-averaged scattered power flow per unit area is given by

$$
\begin{aligned}
\mathbf{S}_{\text {scat }} & =\frac{1}{2} \operatorname{Re}\left\{\mathbf{E}_{\text {scat }} \times \mathbf{H}_{\text {scat }}^{*}\right\} \\
& =\frac{1}{2 Z} \frac{\left|E_{0}\right|^{2}}{r^{2}}\left|\mathcal{F}\left(\mathbf{k}_{\text {in }}, \mathbf{k}_{\text {out }}\right) \cdot \hat{\mathbf{e}}_{\text {in }}\right|^{2} \frac{\mathbf{k}_{\text {out }}}{\mathrm{k}}
\end{aligned}
$$

The scattering cross section $\sigma_{\text {scat }}$ is given by

$$
\sigma_{\text {scat }}=\int \mathrm{d} \Omega_{\text {out }}\left|\mathcal{F}\left(\mathbf{k}_{\text {in }}, \mathbf{k}_{\text {out }}\right) \cdot \hat{\mathbf{e}}_{\text {in }}\right|^{2}
$$

and the total scattered power is

$$
P_{\text {scat }}=\frac{\left|E_{0}\right|^{2}}{2 Z} \sigma_{\text {scat }}
$$

The extinction cross section is related to the imaginary part of the amplitude dyadic in the forward direction as

$$
\sigma_{\text {ext }}=\frac{4 \pi}{k} \operatorname{Im}\left\{\hat{\mathbf{e}}_{\text {in }} \cdot \mathcal{F}\left(\mathbf{k}_{\text {in }}, \mathbf{k}_{\text {in }}\right) \cdot \hat{\mathbf{e}}_{\text {in }}\right\}
$$

In our case, the scattered field is given by the sum of the contributions of the dipolar fields $p_{y}, m_{x}$, and $p_{z}$ given by eqs 3 and 4. In the far field $|\mathbf{r}| \gg\left|\mathbf{r}_{j}\right|$, we have $k\left|\mathbf{r}-\mathbf{r}_{j}\right| \approx k r-\mathbf{k}_{\text {out }} \cdot \mathbf{r}_{j}$ with $\mathbf{r}_{1}=-\mathbf{r}_{2}=(D / 2) \hat{\mathbf{e}}_{y} ; \mathbf{k}_{\text {out }}=k \mathbf{r} / r ; \mathbf{k}_{\text {in }}=k \hat{\mathbf{e}}_{z} ; \hat{\mathbf{e}}_{\text {in }}=\hat{\mathbf{e}}_{y} ;$ and

$$
\begin{aligned}
& k^{2} \overleftrightarrow{\mathbf{G}}_{\mathrm{E}}\left(\mathbf{r}-\mathbf{r}_{j}\right) \mathbf{p}_{j} \approx \frac{e^{i k r}}{4 \pi r}\left\{k^{2} \mathbf{p}_{j}-\left(\mathbf{k}_{\text {out }} \cdot \mathbf{p}_{j}\right) \mathbf{k}_{\text {out }}\right\} e^{-i \mathbf{k}_{\text {out }} \mathbf{r}_{j}} \\
& =\frac{e^{i k r}}{4 \pi r}\left\{\left(\mathbf{k}_{\text {out }} \times \mathbf{p}_{j}\right) \times \mathbf{k}_{\text {out }}\right\} e^{-i \mathbf{k}_{\text {out }} \cdot \mathbf{r}_{j}} \\
& k^{2} \overleftrightarrow{\mathbf{G}}_{\mathrm{M}}\left(\mathbf{r}-\mathbf{r}_{j}\right) \mathbf{p}_{j} \approx i k \frac{e^{i k r}}{4 \pi r}\left\{\mathbf{k}_{\text {out }} \times \mathbf{p}_{j}\right\} e^{-i \mathbf{k}_{\text {out }} \cdot \mathbf{r}_{j}}
\end{aligned}
$$

The extinction cross section is then given by

$$
\begin{aligned}
& \sigma_{\mathrm{ext}}=\frac{4 \pi}{k E_{0}} \operatorname{Im}\left(2 \frac{k^{2}}{4 \pi \varepsilon_{0} \varepsilon_{h}} p_{1 y}-2 Z \frac{k^{2}}{4 \pi} m_{1 x}\right) \\
& =2 k \operatorname{Im}\left\{\tilde{\alpha}_{\mathrm{e} y}+\tilde{\alpha}_{\mathrm{m} x}+\left(\tilde{\alpha}_{\mathrm{e}-\mathrm{m}} k^{2} g_{z x}\right) \tilde{\alpha}_{\mathrm{m} x}\right\}
\end{aligned}
$$

This expression allows the understanding of the main spectral features of a dielectric gap antenna in terms of the simple dipoles induced at each particle. We first notice that the extinction (proportional to the work done by the external field) is associated to the excitation of the electric dipoles along the $y$ axis $\left(2 k \operatorname{Im}\left\{\tilde{\alpha}_{\mathrm{ey}}\right\}\right)$ and the magnetic dipole along the $x$-axis. There is no work done by the external field on the electric dipole along the $z$-axis because they are orthogonal. The energy taken from the excitation of the magnetic dipole is distributed in two terms. The first, $2 k \operatorname{Im}\left\{\tilde{\alpha}_{\mathrm{m} x}\right\}$, corresponds to the excitation of the pure magnetic modes of the particles. The second term, $2 k \operatorname{Im}\left\{\left(\tilde{\alpha}_{\mathrm{e}-\mathrm{m}} k^{2} g_{z x}\right) \tilde{\alpha}_{\mathrm{m} x}\right\}$ is associated to the coupling between the magnetic mode (along the $x$-axis) and the induced electric mode along the $z$-axis $\left(g_{z x}\right)$.

We plot in Figure 6 the result of eq 49 in two different dimers showing small separation (strong coupling regime) and relatively large separation distance (weak coupling regime), 10 and $50 \mathrm{~nm}$, respectively. The far-field spectra, calculated using the theoretical model based on the dipole-dipole interaction formalism, agrees very well both quantitatively and qualitatively 
(a)

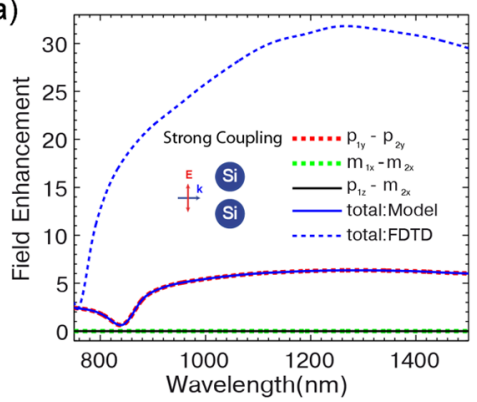

(b)

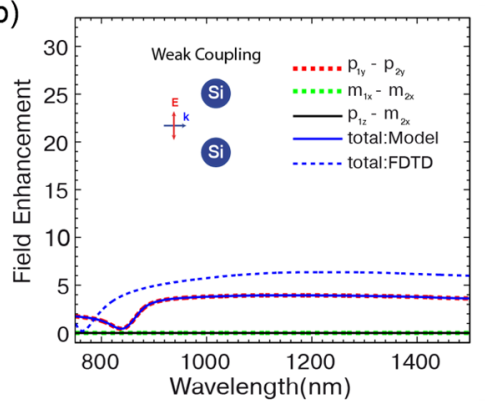

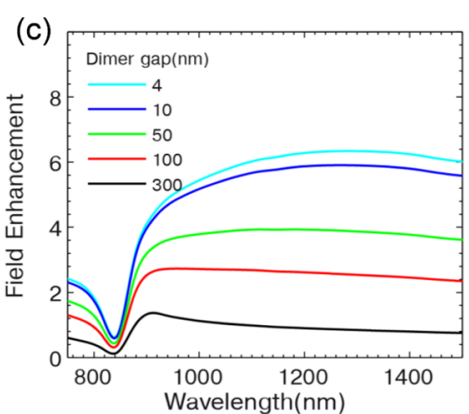

Figure 7. Near-field enhancement spectra calculated using the dipole-dipole model in the strong (a) and in the weak (b) interaction regimes corresponding to gap separations of 10 and $50 \mathrm{~nm}$, respectively (c) Near-field enhancement spectra obtained for a broader range of dimer separations under the same dipole-dipole approximation used in (a) and (b).

with the full numerical electrodynamical calculations performed using the FDTD method (Figure 2a). The first approach allows us to calculate separately the contribution of each interaction to the total extinction of the silicon dimer. Since the theoretical model is based on dipole-dipole interaction, the quadrupolar contribution around $800 \mathrm{~nm}$ obtained in the FDTD calculations is not present in the results obtained with the simple model. The feature around $900 \mathrm{~nm}$ in the weak coupling regime (Figure $6 \mathrm{~b}$ ) can be identified as the electric dipoleelectric dipole (red dashed curve) interaction given by the first term in eq 49. The green dashed curves represent the interaction between the two magnetic dipoles in the two silicon spheres (the second term in eq 49). The black curve is the result of the interaction between the near field induced by the electric and magnetic dipoles in both spheres given by the final term in eq 49. It can be noted that the near-field-induced electric dipole-magnetic dipole interaction provides a substantial contribution to the total extinction when the dimer gap is very small (strong coupling regime), whereas this contribution is not so important at large separation distances (weak coupling regime). This is because the strength of the near field decreases rapidly as the particles move apart. This contribution to the extinction spectra is not observed in the case of metallic nanoparticles since they do not support the magnetic dipolar resonance due to the high losses they present. For completeness and to show the broad range validity of the model, we show in Figure $6 \mathrm{c}$ the far-field spectra obtained within the dipole-dipole model for silicon dimers for a broader range of dimer separations, obtaining good agreement with the FDTD results shown in Figure 2a.

Near-Field Calculations. To understand the interactions that contribute to the total near-field enhancement in the gap, we calculated the near-field spectra in the middle of the gap using the dipolar model. The results are summarized in Figure 7 for both the strong (Figure 7a) and the weak (Figure 7b) interaction regimes. The comparison of the electric field enhancement at the gap of the dimer given by the dipole model (blue) with that obtained within the FDTD full electrodynamical calculations (dashed blue) in Figure 7a shows the limitations of the simple dipole-dipole model when it comes to describing strong localized fields given by evanescent (nonpropagating) components. Opposite to the scattering properties given by propagating far fields, the distribution of near fields often includes many high-order components that contain the evanescent nature of the fields in the proximity of the particles. This local distribution of fields needs to adapt to the particular size of the particle and boundary geometry. In our case, when the gap size is relatively large (weak coupling regime), the polarization of each particle can be correctly approximated by that of a homogeneously polarized sphere, thus the fields can be well described within the dipolar approximation. This situation is clearly illustrated in Figure $7 \mathrm{~b}$ where we compare the results of the near-field enhancement spectra from full electrodynamical calculations (dashed blue line) with those obtained from the dipole-dipole approximation (solid blue line) for a gap separation of $50 \mathrm{~nm}$. As shown in the figure, a reasonable agreement is obtained in this case. However, in the strong coupling regime (Figure 7a), the polarization distribution in the proximity of the surface boundaries becomes highly inhomogeneous due to the presence of the other particle. The description of these inhomogeneities thus cannot be described by the simple dipolar approximation and requires considering much higher-order terms. The comparison between the result of the exact electrodynamical calculation (dashed blue line) and that of the dipolar approximation (solid blue line) shows these limitations clearly. Figure $7 \mathrm{c}$ shows how the contribution of the dipolar-dipolar interaction to the near-field does not change substantially when decreasing the gap size, whereas the exact near-field should increase dramatically for small gap separations (compare, for example, the differences as the gap decreases between the exact results of the near-field in Figure $3 a$ with those approximated ones in Figure 7c).

\section{CONTROL OF SINGLE EMITTERS}

Dielectric antennas present a unique structure to enhance the decay properties of single emitters. ${ }^{8}$ We thus discuss the enhanced fluorescence of a dipolar emitter placed in the gap between our dimer. The decay rate of such an emitter located in the gap is modified due to two competitive processes: transfer of the energy from the emitter to the nanoparticle (observed exclusively for lossy nanoparticles) $)^{32,43}$ and the coupling of the field originated by the emitter to the outgoing radiation. ${ }^{44,45}$ The resulting modification of the emitter's decay rate, known as the Purcell effect, ${ }^{33,34,46}$ has been extensively studied and applied to designing efficient nanoantennas for single photon emission, ${ }^{31}$ enhancement of the fluorescence intensity, ${ }^{32,47,48}$ and emission directivity. ${ }^{45}$ Here, we consider our silicon dimer system as a near-IR antenna and compare its efficiency to that of a plasmonic dimer. To this end, we place electric and magnetic dipolar emitters, which model the dipolar transition moment of a fluorescent molecule, with an intrinsic quantum efficiency of 1 and the decay rate $\Gamma_{0}$, in the geometric center of the dimer and calculate both the power radiated by the system and the dissipation of the energy originated by the 
(a)

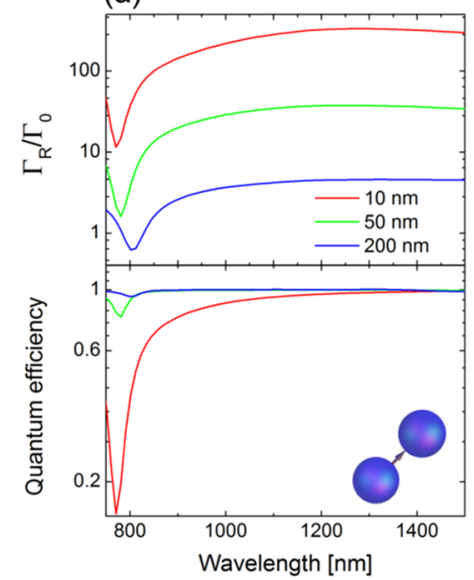

(b)

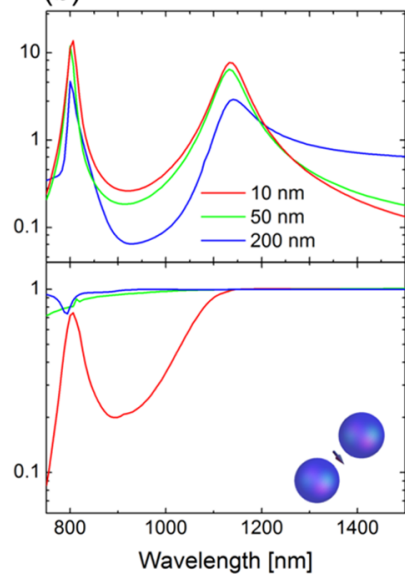

(c)

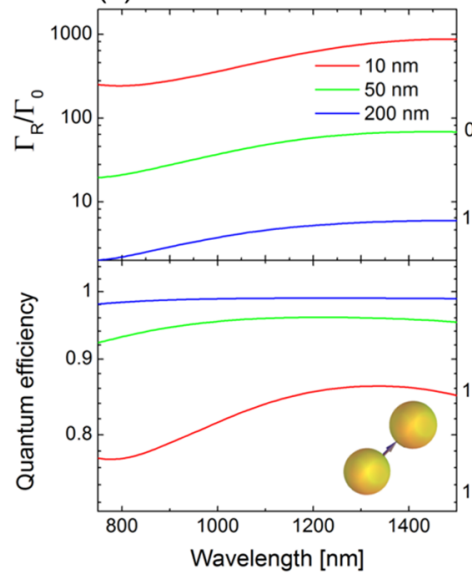

(d)

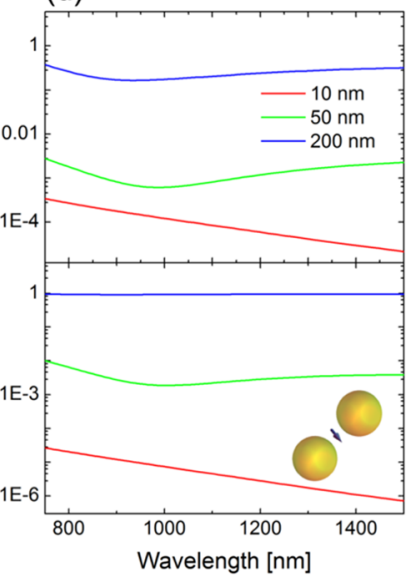

Figure 8. Enhancement of the radiative decay rate and quantum efficiency of an electric dipolar emitter positioned in between two silicon ((a),(b)) and gold $((\mathrm{c}),(\mathrm{d}))$ nanospheres of $150 \mathrm{~nm}$ radius. Orientations of the emitters positioned at the centers of the systems are shown in the schematics. Gap widths are given in the legends.

(a)

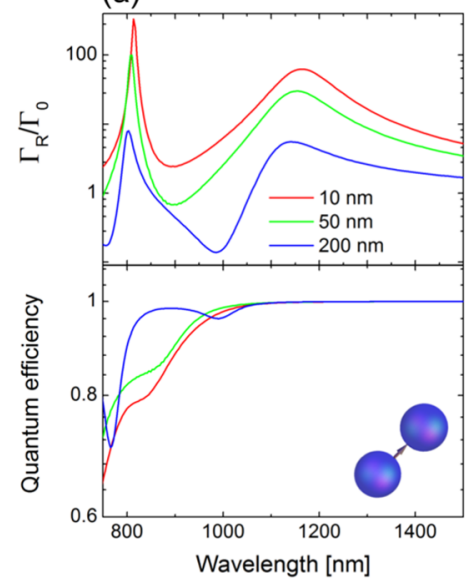

(b)

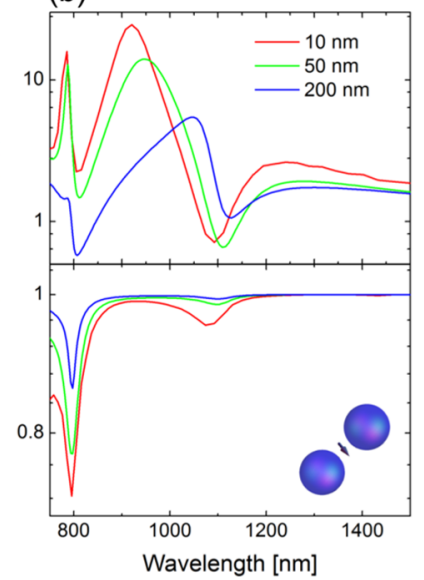

(c)

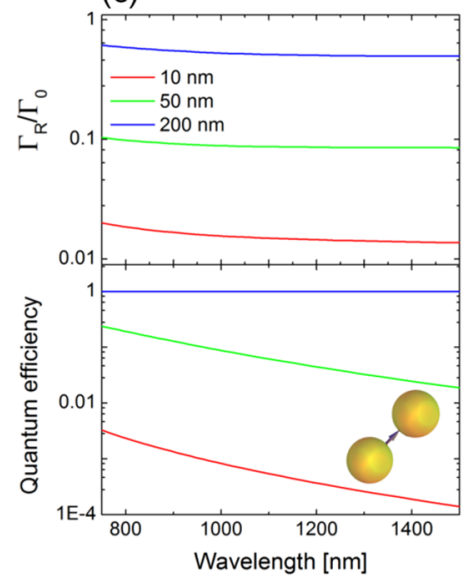

(d)

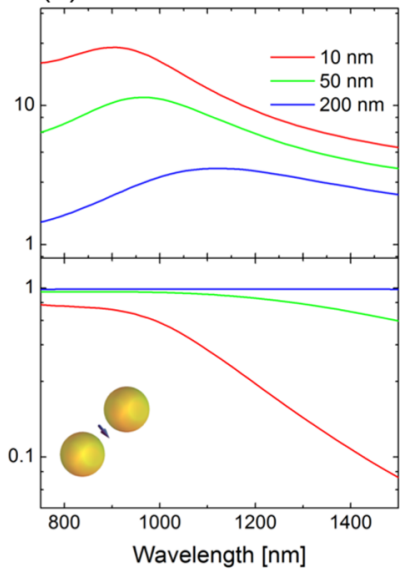

Figure 9. Enhancement of the radiative decay rate and quantum efficiency of a magnetic dipolar emitter positioned in between two silicon ((a),(b)) and gold $((\mathrm{c}),(\mathrm{d}))$ nanospheres of $150 \mathrm{~nm}$ radius. Orientations of the emitters positioned at the centers of the systems are shown in the schematics. Gap widths are given in the legends.

emitter into Ohmic losses. These quantities, when normalized to the power emitted in the absence of the antenna, yield the enhancement of the emitter's radiative decay rate $\Gamma_{\mathrm{R}} / \Gamma_{0}$ and of the nonradiative processes $\Gamma_{\mathrm{NR}} / \Gamma_{0}$. The quantum efficiency is then defined as $Q=\Gamma_{\mathrm{R}} /\left(\Gamma_{\mathrm{R}}+\Gamma_{\mathrm{NR}}\right)$.

In Figure 8 we present the spectra of the radiative decay rate enhancement $\Gamma_{\mathrm{R}} / \Gamma_{0}$ and the quantum efficiencies $Q$ for the electric dipolar emitters, with gap widths of 10, 50, and $200 \mathrm{~nm}$. The emitters are positioned in the geometric centers of the dimers comprising two silicon (Figures $8 \mathrm{a}$ and $8 \mathrm{~b}$ ) or gold (Figures $8 \mathrm{c}$ and $8 \mathrm{~d}$ ) nanospheres, oriented either along (8a and $8 \mathrm{c}$ ) or perpendicularly ( $8 \mathrm{~b}$ and $8 \mathrm{~d}$ ) to the symmetry axis, as shown in the corresponding schematics. The radii of the spheres are $150 \mathrm{~nm}$. Figure 9 represents the same quantities for an analog situation where the emitter is now a magnetic dipole positioned in the gap of the dimer.

Spectra of the radiative enhancement rates of the electric dipolar emitter shown in Figure $8 \mathrm{a}$ and $8 \mathrm{~b}$ exhibit a selectivity of coupling to the geometric resonances similar to that observed for an emitter near a single sphere, ${ }^{8}$ with the orientation along the symmetry axis (Figure 8a) promoting coupling to the electric modes and-in the perpendicular orientation (Figure $8 \mathrm{~b}$ ) - primarily to the magnetic modes. For the smallest gap widths (red lines), at resonance wavelengths the radiative decay rate is enhanced by up to 300 (Figure $8 \mathrm{a}, \lambda$ $=1300 \mathrm{~nm}$ ) and 12 (Figure $8 \mathrm{~b}, \lambda=800 \mathrm{~nm}$ ), depending on the emitter's orientation, while retaining quantum efficiencies over 0.1 . In the setup comprising the gold dimer (Figures $8 \mathrm{c}$ and 8d), we observe a dramatically different behavior for the two orientations of the emitter. When parallel to the symmetry axis (Figure 8c), the dipolar emitter exhibits enhancement of the radiative decay rates up to $1000(\lambda=1500 \mathrm{~nm}$; owing to the super-radiant coherent superposition of the dipoles induced in the spheres), with high quantum efficiency. For the emitter oriented perpendicularly (Figure $8 \mathrm{~d}$ ), the fluorescence is strongly quenched $\left(Q<10^{-5}\right)$.

Magnetic counterparts of the electric dipolar emittersmagnetic dipoles-have recently been investigated as prospective probes of the magnetic components of light or the magnetic local density of states (m-LDOS). ${ }^{49-51}$ It was also shown that, by accessing the magnetic resonances in single dielectric nanoantennas, we obtain a versatile control of the $\mathrm{m}$ LDOS and thus of the radiative properties of such emitters. ${ }^{8,30}$ To extend this notion toward more complex systems, in Figure 
9 we present the enhancement factors and quantum efficiencies of the magnetic dipolar emitter coupled to the dimer silicon and gold antennas. For the emitter oriented along the symmetry axis of the dimer dielectric antenna (Figure 9a), we obtain a strong enhancement of the radiation rate (up to 250 for $10 \mathrm{~nm}$ separation) due to coupling to the magnetic excitations in the antennas. Significantly weaker enhancements (up to 25) are obtained for the emitter oriented perpendicularly to the dimer axis (Figure $9 \mathrm{~b}$ ), as a result of the interplay of the electric and magnetic excitations. The radiation rate from the magnetic emitters in the plasmonic antennas is either strongly quenched and suppressed (Figure 9c) or weakly enhanced (Figure 9d) for the emitter parallel or perpendicular to the dimer axis, respectively.

Similarly as for the electric dipolar emitter, the spectral features of the radiative rate enhancements of the magnetic dipolar emitter, collected in the upper panels of Figure 9a and $9 \mathrm{~b}$, qualitatively resemble those obtained for the magnetic dipolar emitter positioned over a single silicon spherical antenna. ${ }^{8}$ The emission rates for a magnetic dipole in the dimer gap and oriented along the dimer axis (Figure 9a) strongly correlate with the above-mentioned field enhancement for s-polarized light (Figure 3c). As in the case of a single silicon sphere, the dipole preferentially couples with the resonant (quadrupolar- $b_{2}$ - and dipolar- $b_{1}$ ) magnetic modes. However, when the magnetic emitter is oriented perpendicularly to the dimer axis, the field enhancements (and the emission rates) are substantially lower. Moreover, in this case, similarly as for the single silicon sphere (see Figure 1 in ref 8 ), the radiation weakly couples to all electric and magnetic dimer modes.

The quantum efficiency of the emitter is primarily governed by the extinction coefficient of silicon, which grows steadily for energies above around $1 \mathrm{eV}$. Furthermore, significant dips in the quantum efficiency can be qualitatively assigned to the dips in the radiative rate enhancement.

It is worth mentioning that in some frequency ranges the emission rates, for both electric (Figure $8 \mathrm{~b}$ ) and magnetic (Figure 9a) emitters, are strongly reduced. For very small gap widths, this suppression of radiative decays coincides with a low quantum efficiency. However, inhibition of spontaneous emission does not necessarily correlate with quantum efficiency as it can be seen from our results for large gap widths. Notice that emission rates are constrained by specific sum rules derived from a Kramers-Kronig relation. ${ }^{52,53}$ In particular, any reduction in spontaneous emission rate over some range of wavelengths must necessarily be compensated by increases over some other range of frequencies. The strong dip in the emission rates observed in Figure $8 b$ (for a perpendicular electric dipole) and 9a (for a parallel magnetic dipole) could also be observed even for a single lossless sphere, where quantum efficiency is 1 , as discussed in ref 8 . These results open new intriguing possibilities for applications requiring inhibition of spontaneous emission for specific dipole orientations.

Low losses and reasonably high values of the enhancement factors obtained for the dielectric dimers, in comparison to the factors obtained in plasmonic antennas, as well as the ability to tune the position of the resonances highlight the benefits of using dielectric nanoantennas for enhancing and controlling the emission from the dipolar electric and magnetic emitters.

\section{CONCLUSION}

A full electrodynamical study of the optical response of subwavelength silicon dimers was carried out to exploit their far-field and near-field properties as an antenna for novel fieldenhanced spectroscopies. The interaction between the excited predominant dipolar modes (electric and magnetic) is analyzed by means of an analytical model based on electric and magnetic dipole-dipole interactions, providing a direct understanding of the electrodynamical interactions responsible for the optical properties of the dimer. This simple model not only identifies the spectral features originating from different electric and magnetic interactions but also helps to get insights about the origin of the near-field enhancement in the gap. We have numerically demonstrated that even though the field-enhancing capability of dielectric nanoparticles is comparatively less intense than that provided by metallic particles the absence of quenching of radiative emission and high quantum efficiency shown by an emitting molecule coupled to the electromagnetic modes of a dielectric nanoantenna makes these structures promising building blocks to develop optical magnetism. Together with zero absorption and directional emission, ${ }^{18}$ and the properties shown in this research, dielectric antennas become an alternative to conventional metallic ones for fieldenhanced spectroscopy and sensing, free of resistive losses and with the property of enhancing the electric, the magnetic, or both fields.

\section{AUTHOR INFORMATION}

\section{Corresponding Author}

*E-mail: p.albella@imperial.ac.uk; aizpurua@ehu.es.

\section{Notes}

The authors declare no competing financial interest.

\section{ACKNOWLEDGMENTS}

We acknowledge funding from the ETORTEK project nanoiker of the Department of Industry of the Basque Country, the project of Groups of excellence IT756-13 of the Basque Country Government, and project FIS2010-19609-C02-01 of the Spanish Ministry of Innovation. P. Albella acknowledges the support received from the European Science Foundation for the activity entitled: "New approaches to Biochemical sensing with Plasmonic Nanobiophotonics" (PLASMON-BIONANOSENSE) and from the German Research Council Foundation (DFG). S.A. Maier acknowledges support by the EPSRC and the U.S. Army International Technology Centre Atlantic (USAITC-A) and the Office of Naval Research (ONR and ONR Global). J.J. Saenz acknowledges support from the Spanish Ministerio de Ciencia e Innovacion through Consolider NanoLight (CSD2007-00046) and from the Comunidad de Madrid Microseres-CM (S2009/TIC-1476). F. Moreno acknowledges the support of the Spanish Ministerio de Ciencia e Innovacion through the grant FIS2010-21984.

\section{REFERENCES}

(1) Giannini, V.; Fernández-Domínguez, A. I.; Sonnefraud, Y.; Roschuk, T.; Fernández- García, R.; Maier, S. A. Controlling Light Localization and Light-Matter Interactions with Nanoplasmonics. Small 2010, 6, 2498-507.

(2) Popa, B.-I.; Cummer, S. Compact Dielectric Particles as a Building Block for Low-Loss Magnetic Metamaterials. Phys. Rev. Lett. 2008, 100, 207401. 
(3) Valentine, J.; Zhang, S.; Zentgraf, T.; Ulin-Avila, E.; Genov, D. A.; Bartal, G.; Zhang, X. Three-Dimensional Optical Metamaterial with a Negative Refractive Index. Nature 2008, 455, 376-379.

(4) Kuznetsov, A. I.; Miroshnichenko, A. E.; Fu, Y. H.; Zhang, J.; Luk'yanchuk, B. Magnetic Light. Sci. Rep. 2012, 2, 492.

(5) Miroshnichenko, A. E.; Luk'yanchuk, B.; Maier, S. A.; Kivshar, Y. S. Optically Induced Interaction of Magnetic Moments in Hybrid Metamaterials. ACS Nano 2012, 6, 837-842.

(6) Evlyukhin, A. B.; Reinhardt, C.; Seidel, A.; Luk'yanchuk, B. S.; Chichkov, B. N. Optical response features of Si-nanoparticle arrays. Phys Rev B 2010, 82, 45404.

(7) García-Etxarri, A.; Gómez-Medina, R.; Froufe-Pérez, L. S.; López, C.; Chantada, L.; Scheffold, F.; Aizpurua, J.; Nieto-Vesperinas, M.; Sáenz, J. J. Strong Magnetic Response of Submicron Silicon Particles in the Infrared. Opt. Express 2011, 19, 4815-4826.

(8) Schmidt, M. K.; Esteban, R; Saenz, J. J.; Suárez-Lacalle, I.; Mackowski, S.; Aizpurua, J. Dielectric Antennas - a Suitable Platform for Controlling Magnetic Dipolar Emission. Opt. Express 2012, 20, 13636-13650.

(9) Evlyukhin, A. B.; Novikov, S. M.; Zywietz, U.; Eriksen, R. L.; Reinhardt, C.; Bozhevolnyi, S. I.; Chichkov, B. N. Demonstration of Magnetic Dipole Resonances of Dielectric Nanospheres in the Visible Region. Nano Lett. 2012, 12, 3749.

(10) Mühlschlegel, P.; Eisler, H. J.; Martin, O. J. F.; Hecht, B.; Pohl, D. W. Resonant Optical Antennas. Science 2005, 308, 1607-1609.

(11) Hillenbrand, R; Taubner, T.; Keilmann, F. Phonon-Enhanced Light Matter Interaction at the Nanometre Scale. Nature 2002, 418, $159-162$.

(12) García-Cámara, B.; González, F.; Moreno, F. Linear Polarization Degree for Detecting Magnetic Properties of Small Particles. Opt. Lett. 2010, 35, 4084-4086.

(13) Gómez-Medina, R.; García-Cámara, B.; Suárez-Lacalle, I.; González, F.; Moreno, F.; Nieto-Vesperinas, M.; Sáenz, J. J. Electric and Magnetic Dipolar Response of Germanium Nanospheres: Interference Effects, Scattering Anisotropy, And Optical Forces. J. Nanophotonics 2011, 5, 053512.

(14) Devilez, A.; Stout, B.; Bonod, N. Compact Metallo-Dielectric Optical Antenna for Ultra Directional and Enhanced Radiative Emission. ACS Nano 2010, 4, 3390-3396.

(15) Nieto-Vesperinas, M.; Gómez-Medina, R.; Sáenz, J. J. AngleSuppressed Scattering and Optical Forces on Submicrometer Dielectric Particles. J. Opt. Soc. Am. A 2011, 28, 54-60.

(16) Kerker, M.; Wang, D.; Giles, C. L. Electromagnetic Scattering by Magnetic Spheres. J. Opt. Soc. Am. 1983, 73, 765-767.

(17) Rolly, B.; Stout, B.; Bonod, N. Boosting the Directivity of Optical Antennas with Magnetic and Electric Dipolar Resonant Particles. Opt. Express 2012, 20, 20376-20386.

(18) Geffrin, J. M.; García-Cámara, B.; Gómez-Medina, R.; Albella, P.; Froufe-Pérez, L. S.; Eyraud, C.; Litman, A.; Vaillon, R.; González, F.; Nieto-Vesperinas, M.; Sáenz, J. J.; Moreno, F. Magnetic and Electric Coherence in Forward- And Back-Scattered Electromagnetic Waves by a Single Dielectric Subwavelength Sphere. Nat. Commun. 2012, 3, 1171.

(19) Person, S.; Jain, M.; Lapin, Z.; Sáenz, J. J.; Wicks, G.; Novotny, L. Demonstration of Zero Optical Backscattering from Single Nanoparticles. Nano Lett. 2013, 3-6.

(20) Fu, Y. H.; Kuznetsov, A. I.; Miroshnichenko, A. E.; Yu, Y. F.; Luk'yanchuk, B. Directional Visible Light Scattering by Silicon Nanoparticles. Nat. Commun. 2013, 4, 1527.

(21) Nieto-Vesperinas, M.; Sáenz, J. J.; Gómez-Medina, R.; Chantada, L. Optical Forces on Small Magnetodielectric Particles. Opt. Express 2010, 18, 11428-43.

(22) Shi, L.; Xifré-Pérez, E.; García de Abajo, F. J.; Meseguer, F. Looking through the Mirror: Optical Microcavity-Mirror Image Photonic Interaction. Opt. Express 2012, 20, 11247-55.

(23) Lagos, N.; Sigalas, M. Single Particle Detection in a System of Two Microdisks. Sens. Actuators, B 2011, 153, 252-255.

(24) Twersky, V. Multiple Scattering of ElectromagneticWaves by Arbitrary Configurations. J. Math. Phys. 1967, 8, 589.
(25) Bruning, J. H.; Lo, Y. T. Multiple Scattering of EM Waves by Spheres Part I-Multipole Expansion and. IEEE Trans. Antennas Propag. 1971, 19, 378-390.

(26) Fuller, K. A. Optical Resonances and Two-Sphere Systems. Appl. Opt. 1991, 30, 4716-31.

(27) Miyazaki, H.; Jimba, Y. Ab Initio Tight-Binding Description of Morphology-Dependent Resonance in a Bisphere. Phys. Rev. B 2000, 62, 7976-7997.

(28) Spinelli, P.; Verschuuren, M. A.; Polman, A. Broadband Omnidirectional Antireflection Coating Based on Subwavelength Surface Mie Resonators. Nat. Commun. 2012, 3, 692.

(29) Chen, X.-W.; Agio, M.; Sandoghdar, V. Metallodielectric Hybrid Antennas for Ultrastrong Enhancement of Spontaneous Emission. Phys. Rev. Lett. 2012, 108, 233001.

(30) Rolly, B.; Bebey, B.; Bidault, S.; Stout, B.; Bonod, N. Promoting Magnetic Dipolar Transition in Trivalent Lanthanide Ions with Lossless Mie Resonances. Phys. Rev. B 2012, 85, 245432.

(31) Novotny, L.; van Hulst, N. Antennas for Light. Nat. Photonics 2011, 5, 83-90.

(32) Mackowski, S.; Wörmke, S.; Maier, A. J.; Brotosudarmo, T. H. P.; Harutyunyan, H.; Hartschuh, A.; Govorov, A. O.; Scheer, H.; Bräuchle, C. Metal-Enhanced Fluorescence of Chlorophylls in Single Light-Harvesting Complexes. Nano Lett. 2008, 8, 558-64.

(33) Purcell, E. Spontaneous Emission Probabilities at Radio Frequencies. Phys. Rev. 1946, 69, 681.

(34) Koenderink, A. F. On the Use of Purcell Factors for Plasmon Antennas. Opt. Lett. 2010, 35, 4208-10.

(35) Novotny, L.; Hecht, B. Principles of Nano-Optics; Cambridge University Press: Cambridge, England, 2006.

(36) Palik, E. D. Handbook of Optical Constants of Solids; Academic Press: New York, 1997.

(37) Taflove, A. Application of the Finite-Difference Time-Domain Method to Sinusoidal Steady-State Electromagnetic-Penetration Problems. IEEE Trans. Electromagn. Compat. 1980, EMC-22, 191-202.

(38) Rechberger, W.; Hohenau, A.; Leitner, A.; Krenn, J.; Lamprecht, B.; Aussenegg, F. Optical Properties of Two Interacting Gold Nanoparticles. Opt. Commun. 2003, 220, 137-141.

(39) Ru, E. C. L.; Blackie, E.; Meyer, M.; Etchegoin, P. G. Surface Enhanced Raman Scattering Enhancement Factors: A Comprehensive Study. J. Phys. Chem. C 2007, 111, 13794-13803.

(40) Bohren, C. F.; Huffman, D. R. Absorption and Scattering of Light by Small Particles; John Wiley and Sons: New York, 1983.

(41) Tai, C.-T. Dyadic Green Functions in Electromagnetic Theory, 2nd ed.; IEEE Press: New York, 1993.

(42) Jackson, J. D. Classical Electrodynamics, 3rd ed.; John Wiley and Sons: New York, 1999.

(43) Anger, P.; Bharadwaj, P.; Novotny, L. Enhancement and Quenching of Single-Molecule Fluorescence. Phys. Rev. Lett. 2006, 96, 113002 .

(44) Ruppin, R. Decay of an Excited Molecule near a Small Metal Sphere. J. Chem. Phys. 1982, 76, 1681.

(45) Curto, A. G.; Volpe, G.; Taminiau, T. H.; Kreuzer, M. P.; Quidant, R.; van Hulst, N. F. Unidirectional Emission of a Quantum Dot Coupled to a Nanoantenna. Science 2010, 329, 930-933.

(46) Froufe-Pérez, L. S.; Carminati, R.; Sáenz, J. J. Fluorescence Decay Rate Statistics of a Single Molecule in a Disordered Cluster of Nanoparticles. Phys. Rev. A 2007, 76, 013835.

(47) Laroche, M.; Albaladejo, S.; Carminati, R.; Sáenz, J. J. Optical Resonances in One-Dimensional Dielectric Nanorod Arrays: FieldInduced Fluorescence Enhancement. Opt. Lett. 2007, 32, 2762-2764.

(48) Aroca, R. F.; Teo, G. Y.; Mohan, H.; Guerrero, A. R.; Albella, P.; Moreno, F. Plasmon-Enhanced Fluorescence and Spectral Modification in SHINEF. J. Phys. Chem. C 2011, 115, 20419-20424.

(49) Karaveli, S.; Zia, R. Spectral Tuning by Selective Enhancement of Electric and Magnetic Dipole Emission. Phys. Rev. Lett. 2011, 106, 193004.

(50) Dodson, C. M.; Zia, R. Magnetic Dipole and Electric Quadrupole Transitions in the Trivalent Lanthanide Series: Calculated 
Emission Rates and Oscillator Strengths. Phys. Rev. B 2012, 86, 125102.

(51) Taminiau, T. H.; Karaveli, S.; van Hulst, N. F.; Zia, R. Quantifying the Magnetic Nature of Light Emission. Nat. Commun. 2012, 3, 979.

(52) Barnett, S.; Loudon, R. Sum Rule for Modified Spontaneous Emission Rates. Phys. Rev. Lett. 1996, 77, 2444-2446.

(53) Carminati, R.; Sáenz, J. J. Density of States and Extinction Mean Free Path of Waves in Random Media: Dispersion Relations and Sum Rules. Phys. Rev. Lett. 2009, 102, 093902. 